Cite this: J. Mater. Chem. C, 2014, 2 , 6056

Received 15th April 2014

Accepted 10th June 2014

DOI: $10.1039 / \mathrm{c} 4 \mathrm{tc0} 0759 \mathrm{j}$

www.rsc.org/MaterialsC

\section{Controlling templating effects at the organic/ inorganic interface using (111) oriented copper iodide $\uparrow$}

\begin{abstract}
L. A. Rochford, ${ }^{\star a}$ D. S. Keeble, ${ }^{\text {b }}$ O. J. Holmes, ${ }^{a}$ G. J. Clarkson ${ }^{a}$ and T. S. Jones ${ }^{a}$
Structural templating of organic semiconductors affords control of out-of-plane film structure and molecular orientation with respect to solid surfaces. Herein we use a prototypical copper iodide (Cul)/ planar phthalocyanine system to produce detailed surface and crystal structure information using atomic force microscopy (AFM) and X-ray diffraction (XRD). The out-of-plane structure of the Cul layer was characterised and identified as the (111) plane of single crystal Cul. The dependance of surface morphology and grain size in the Cul (111) templating layer upon substrate temperature was demonstrated. The formation of a thin film of iron phthalocyanine (FePc) on this model layer was characterised at multiple points during growth, changes in the surface morphology were observed, and the crystal structure of the final film was used to infer the molecular orientation therein. These changes were elucidated using the re-determined single crystal structure of FePc which is also presented.
\end{abstract}

\section{Introduction}

The ability to control the morphology and crystal structure of organic semiconductors allows their physical and electronic properties to be tuned concomitantly.$^{1-3}$ Desirable characteristics for specific device applications including organic photovoltaic (OPV), thin film transistor (OTFT) and light emitting diode (OLED) geometries can be preferentially selected. ${ }^{4,5}$ To this end, thin organic ${ }^{6}$ or inorganic ${ }^{7}$ layers have been employed as structural templates for the improvement of device parameters. A prominent example of this methodology is the insertion of a copper(I) iodide interlayer between a transparent electrode (indium tin oxide) and a planar phthalocyanine (Pc) active layer to modify molecular orientation in the organic film. ${ }^{7}$

The effect of this structural modification has been observed in $\mathrm{OPVs}^{8}$ and theoretical simulations have been used to model the change in charge transport characteristics in phthalocyanine/fullerene devices. ${ }^{9}$ Despite this, the nature of the interaction causing this modification has not been studied in any depth. Planar Pc molecules are widely used in organic electronics and have been used to fabricate a variety of efficient devices. ${ }^{10}$

\footnotetext{
${ }^{a}$ Department of Chemistry, University of Warwick, Gibbet Hill Road, Coventry, CV4 7AL, UK. E-mail: l.rochford@warwick.ac.uk

${ }^{b}$ Department of Physics, University of Warwick, Gibbet Hill Road, Coventry, CV4 7AL, UK

$\dagger$ Electronic supplementary information (ESI) available: Additional XRD traces of untemplated FePc films, a plot of surface roughness $v s$. FePc thickness, expanded views of a high-angle CuI XRD trace and additional single crystal structure information are included. CCDC 996880. For ESI and crystallographic data in CIF or other electronic format DOI: 10.1039/c4tc00759j
}

The crystal structure of planar Pcs is inherently anisotropic due to face-to-face packing motifs creating short intermolecular distances within stacks and larger inter-stack spacings. ${ }^{11,12}$ This has been shown to impact charge transport in single crystals ${ }^{13}$ and thin films. ${ }^{14}$ Therefore, controlling the direction along which the molecules have closest contacts can lead to improved device characteristics. ${ }^{15}$

We report the preparation and characterisation of evaporated copper iodide (CuI) thin films at both ambient and elevated substrate temperatures. Grain size and morphology in these films was shown to be strongly dependant on substrate temperature during growth and the use of high growth temperature films as structural templates for a planar phthalocyanine $(\mathrm{FePc})$ was explored. Control of both molecular orientation and grain size in FePc films was demonstrated using this methodology. The crystal structure of FePc was also redetermined to enable accurate assignment of the crystal structure to the thin films.

\section{Results and discussion}

Firstly, in order to fully understand the proposed organic molecule templating process, the crystal structure of the $\mathrm{CuI}$ layer prepared at ambient substrate temperatures, as previously shown in the literature, was determined. Evaporated $\mathrm{CuI}$ films of $1 \mathrm{~nm}$ thickness, as used by Rand et al., ${ }^{9}$ were prepared and XRD patterns were recorded in which no CuI peaks were observed. Thicker films $(15 \mathrm{~nm})$ were grown at the same rate $(0.5$ $\AA \mathrm{s}^{-1}$ ), which produced a single broad (as shown in Fig. 1(a)) peak at $2 \theta=25.5^{\circ}$ corresponding to the (111) lattice spacing of CuI (ICSD no. 23 990). Atomic force microscopy (AFM) was used 


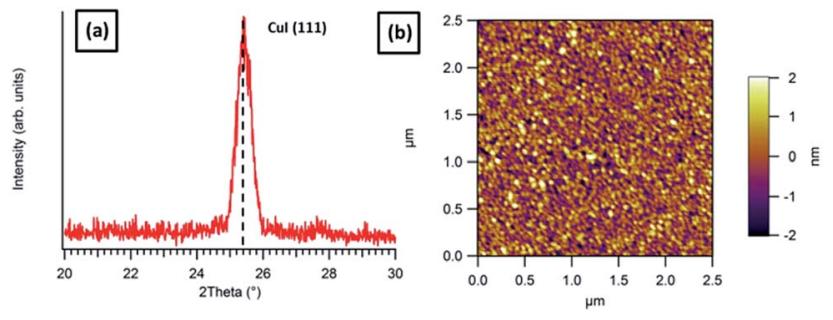

Fig. 1 (a) XRD trace revealing predominantly (111) oriented growth and (b) AFM height image of $15 \mathrm{~nm}$ Cul grown on ambient temperature $\mathrm{SiO}_{2}$.

to observe the morphology of the film, which showed high polycrystallinity (Fig. 1). The films were made up of grains less than $20 \mathrm{~nm}$ in size and exhibited low surface roughness $\left(R_{\mathrm{q}}\right)$ values $(0.7 \mathrm{~nm})$, suggesting that $\mathrm{CuI}$ grows as a highly polycrystalline film with predominantly (111) out-of-plane orientation at room temperature on $\mathrm{SiO}_{2}$.

If the (111) faces present in a highly polycrystalline CuI thin film are responsible for the templating effect, ${ }^{\mathbf{1 9}}$ increasing the size of these faces should allow observation of the nucleation process of an organic layer grown on top. To produce thin films with larger grains more suitable for scanning probe imaging, the substrate temperature was increased to $200{ }^{\circ} \mathrm{C}$. This produced a clear change in morphology; $15 \mathrm{~nm}$ films now consisted of dispersed islands with up to $80 \mathrm{~nm}$ high out-ofplane protrusions and roughly hexagonal shapes in AFM images (Fig. 2(a)). The growth was clearly in the form of a VolmerWeber $^{\mathbf{2 0}}$ (island growth) mode as islands with a variety of sizes and heights were evident. This suggests both that islands are preferentially grown over layer-by-layer formation (no wetting layer was observed), and that islands have nucleated at various points during the growth process. ${ }^{21} \mathrm{~A}$ three dimensional render of the same image is also presented, showing clear faceting and steps in the islands of CuI which suggest that these features are single crystals (Fig. 2(b)).

XRD patterns of the island-like films again showed only (111)-type reflections, indicating that the orientation of the islands is unaltered by the change in growth conditions (a digitally magnified trace showing the (222) and (333) is shown in the ESI, Fig. SI03 $\dagger$ ). Furthermore, the hexagonal habit of the islands suggests 3- or 6-fold planar symmetry, and this is also consistent with the assignment of (111) oriented islands. Additionally, the decreased peak width, and observation of higher angle (111)-type reflections such as the (222) and (333) from the island-like film are both consistent with the assertion from AFM that the islands were mono-crystalline.

The elevated substrate temperature produced highly crystalline CuI islands aligned with their (111) planes parallel to the substrate. This creates a non-uniform surface upon which to grow the organic overlayer, as molecules from the incident molecular beam will impinge on single-crystal $\mathrm{CuI}$ islands, as well as the areas of bare $\mathrm{SiO}_{2}$ substrate between them. As the interaction between the CuI and FePc molecules was the focus of this study a complete coverage of the oriented CuI crystallites was required: to this end films of $45 \mathrm{~nm}$ thickness were grown at the same rate and elevated substrate temperature to produce a continuous film with large (111) grains. This process produced highly crystalline continuous (111) oriented films with grains up to $1 \mu \mathrm{m}$ (observed by AFM, Fig. 2(d)). In the corresponding XRD patterns (Fig. 2(e)) Laue fringes were observed around the (111) peak, confirming the high crystallinity and uniform thickness of the film.

By creating (111) oriented CuI films, uniform surfaces were provided upon which to observe the formation of FePc crystallites on the crystal faces responsible for the templating effect. Successive growth of FePc on to CuI (111) films was carried out in situ without exposure to air to ensure no surface contamination was introduced. FePc films of 5, 10 and $50 \mathrm{~nm}$ thickness were grown after cooling the CuI films to room temperature in vacuum. The thinnest films showed non-uniform nucleation and growth of FePc over the CuI (111) faces, and FePc islands were present in combination with areas of bare CuI substrate (Fig. 3(a)). This is in direct contrast to the behaviour usually observed with ambient substrate temperature growth of planar phtalocyanines on weakly interacting substrates such as glass or $\mathrm{SiO}_{2}{ }^{22}$

In thicker films (10 $\mathrm{nm} \mathrm{FePc})$ the number and size of the FePc grains increased, although CuI grain boundaries were still visible due to non-uniform coverage of the overlayer. In $50 \mathrm{~nm}$ FePc films large grains (up to $1 \mu \mathrm{m}$ longest dimension) were observed and the CuI substrate is no longer visible. The grains were discrete and did not grow into one another as a continuous film, suggesting that they were contained on the individual $\mathrm{CuI}$ (111) faces. Depositing FePc in this way increased the surface roughness of the system: the $5 \mathrm{~nm} \mathrm{FePc} / \mathrm{CuI}$ bilayer had an $R_{\mathrm{q}}$ of $10.2 \mathrm{~nm}$ compared with $2.9 \mathrm{~nm}$ for the bare CuI. Increasing the thickness to $10 \mathrm{~nm}$ increased the roughness further $\left(R_{\mathrm{q}}=15.5\right.$ $\mathrm{nm}$ ) and at $50 \mathrm{~nm}$ the $R_{\mathrm{q}}$ reached a peak of $17.4 \mathrm{~nm}$.

These observations suggest that the FePc layer was also formed via a 3D island growth mode, wherein the roughness initially increases rapidly due to the formation of high aspect ratio islands. These islands grow preferentially out of the surface plane, and appear to saturate at a roughness of around $17.6 \mathrm{~nm}$. Once this roughness was achieved, the high aspect ratio of the islands precludes further growth out-of-plane, and instead promotes growth across the voids between the islands. This behaviour was demonstrated in each of the thicknessdependant AFM images; even in the thinnest film $(5 \mathrm{~nm})$ islands with out-of substrate-plane heights of up to $35 \mathrm{~nm}$ were observed. A plot of roughness against film thickness which shows an approximately exponential increase is included in the ESI, Fig. SI02. $\dagger$

To confirm changes in crystal structure or growth orientation, XRD patterns were recorded from each of the FePc films. The $5 \mathrm{~nm}$ and $10 \mathrm{~nm}$ films yielded no Bragg peaks corresponding to the FePc layer; instead only the strong CuI (111) peak was observed. In the $50 \mathrm{~nm}$ film a peak at $2 \theta=28.1^{\circ}$ was seen (Fig. 3(b)), seemingly in place of any low angle peaks previously observed from FePc films grown on bare $\mathrm{SiO},{ }^{2}$ which were previously indexed as (001) or (100). ${ }^{23}$ When FePc was grown to the same thickness $(50 \mathrm{~nm})$ at both ambient and elevated substrate temperature $\mathrm{SiO}_{2}$ (see ESI, Fig. SI01†) a single 

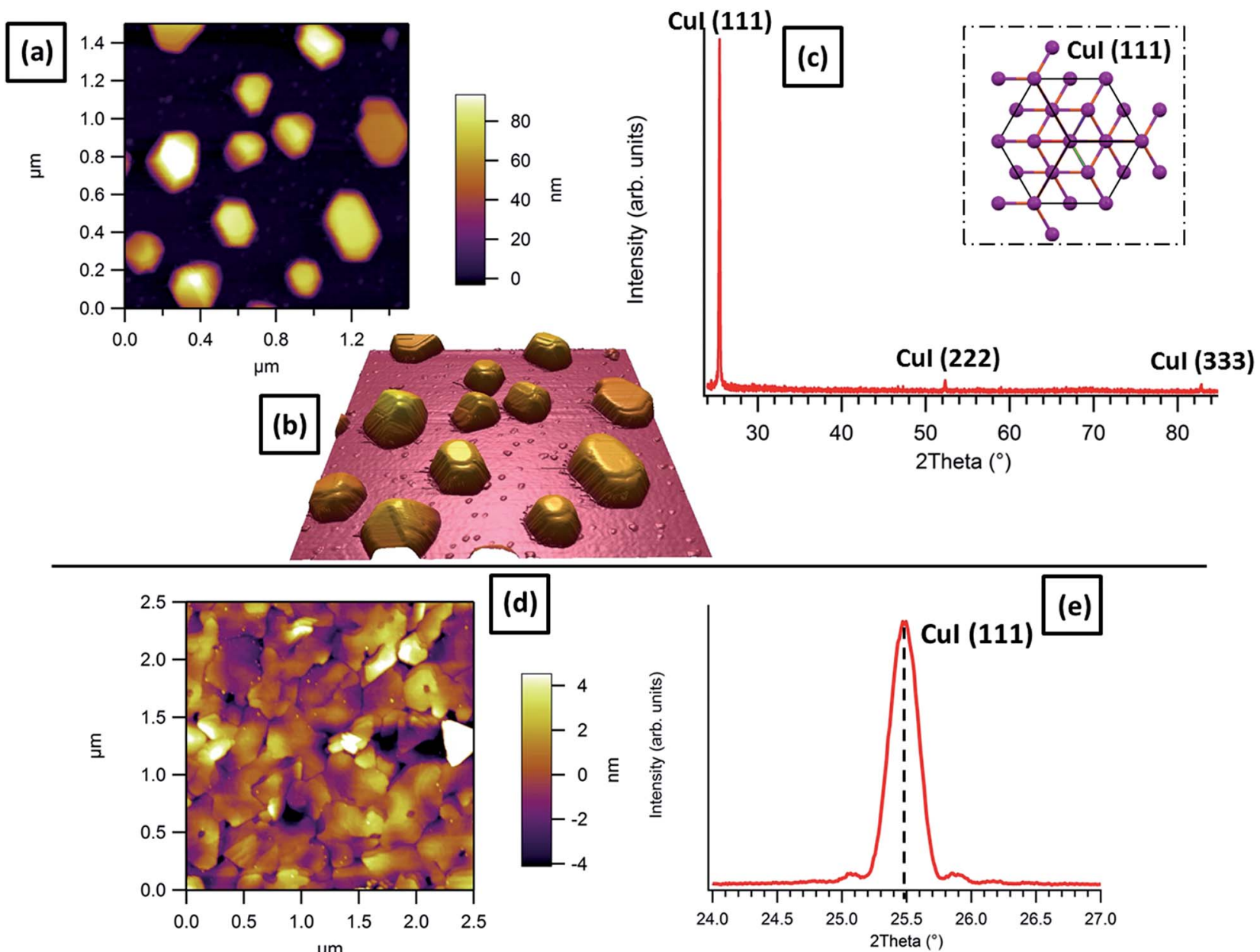

Fig. 2 (a) AFM height image, (b) 3D render of the same image (c) an XRD pattern of a $15 \mathrm{~nm}$ thick Cul/SiO $\mathrm{S}_{2}$ film (inset shows a view orthogonal to the (111) plane). The lower panel shows the (d) AFM height image and (e) XRD pattern for a $45 \mathrm{~nm}$ thick $\mathrm{Cul} / \mathrm{SiO}_{2}$ film.
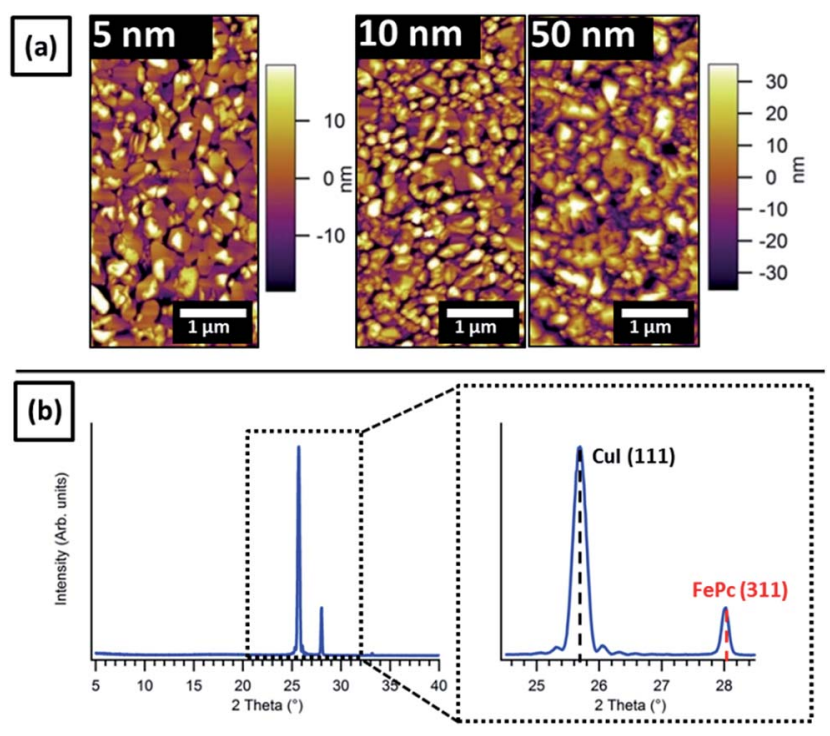

Fig. 3 (a) Series of AFM height images of FePc/Cul bilayers with increasing FePc thickness and (b) the XRD pattern of the $50 \mathrm{~nm}$ thickness $\mathrm{FePc} / \mathrm{Cul} / \mathrm{SiO}_{2}$ film. low angle peak is observed and the higher angle peak is absent. This confirms that the presence of the CuI layer was modifying the growth of the FePc film and producing a change in the outof-plane structure.

In order to ensure the accurate assignment of crystal structure within the films, a high quality single crystal structure of FePc was necessary for comparison. Single crystals of FePc were grown using thermal gradient sublimation in high vacuum. This produced high aspect ratio, needle-like single crystals, the largest of which $(150 \times 75 \times 40 \mu \mathrm{m})$ was used for the singlecrystal XRD experiment for structure determination. The resulting single crystal structure (CCDC no. 996880) has an improved $R$ value and is subtly different from the FePc single crystal data previously published. ${ }^{24}$ These structures are summarised and visualisations of each structure are presented in Fig. 4, while complete structural details are presented in the ESI (Fig. SI04†).

Using this re-determined structure, the peak at $28.1^{\circ}$ now corresponds to the (311) plane, which is close to one of the herringbone stacked molecular planes (Fig.4 (a)). As reflection geometry (as utilised here) is most sensitive to out-of-plane reflections (i.e. the diffraction vector $\overrightarrow{\boldsymbol{d}^{*}}$ is always normal to the 


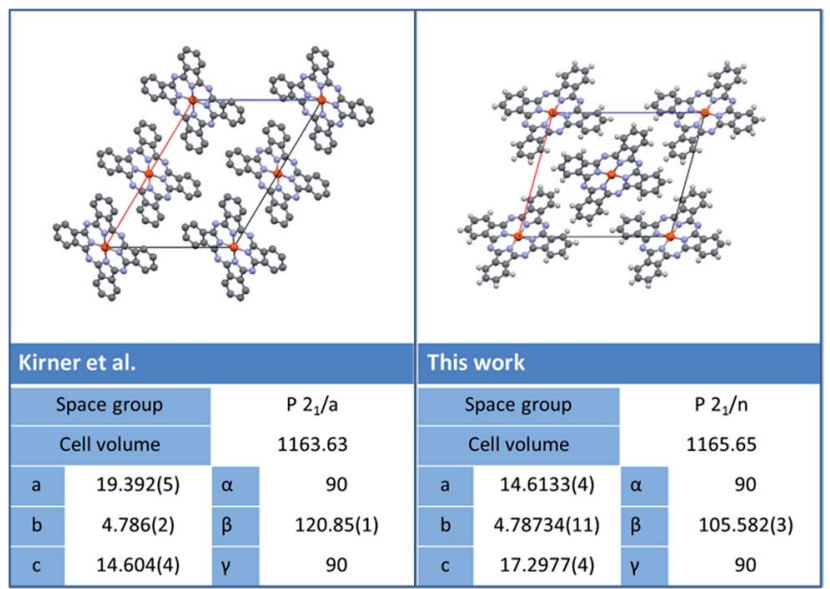

Fig. 4 - Comparison of the previously published FePc single crystal structure and our redetermination, upper panels show a view down the ' $b$ ' axis and parameters are tabulated underneath.

surface), this suggests that the (311) plane is close to parallel to the substrate and therefore the CuI (111) surface.

This implies a molecular orientation in some stacks which is flat-lying with respect to the underlying $\mathrm{SiO}_{2}$ and $\mathrm{CuI}(111)$ layer, with the FePc $\pi$ system projecting out of the CuI (111) plane. Increasing the substrate temperature increases the size of the CuI grains of which the film was composed, and due to the uniform out-of-plane orientation across the film this produces larger CuI (111) faces. These faces act as a structural template for the impinging FePc molecules, both controlling the relative orientation of the molecules and increasing the size of the grains by controlling nucleation. Using our re-determined crystal structure the change in orientation can be elucidated as the transition from all molecules edge-on ((101) oriented) to inclusion of face-on ((311) oriented) stacks with respect to the substrate plane (Fig. 5). In both cases (with and without CuI) the single diffraction peaks used to ascertain this could be indexed using the same single crystal structure. This leads us to the conclusion that although the orientation of the molecules with respect to the substrate was altered the crystal structure was defined by the same unit cell.
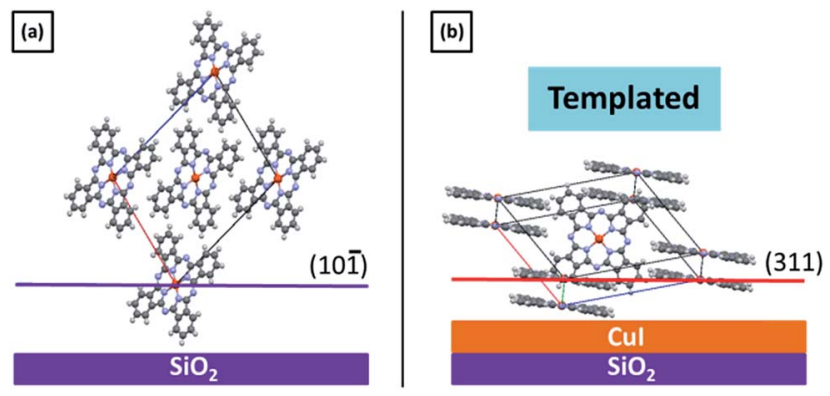

Fig. 5 - Relative molecular orientation of FePc on (a) bare $\mathrm{SiO}_{2}$ and (b) a Cul templating layer. The same unit cell is shown in both cases and planes responsible for the peaks observed in XRD indicated with coloured lines and labelled.
If the crystal structure of the FePc was unchanged and the (311) plane was completely parallel to the surface an inherent inclination of the molecular plane with respect to the surface would be present at the interface. This is due to the facts that the (311) plane is not perfectly parallel to the molecular plane and the FePc molecule is slightly distorted in the single crystal. However, the diffractometer and experimental geometry used here will detect diffraction from planes close to as well as at surface normal. In this case a situation where the (311) plane was projected out of the surface plane at a slight angle (away from surface normal) and a situation where the molecule is lying flat on top of the $\mathrm{CuI} / \mathrm{SiO}_{2}$ layer would produce very similar diffraction features. However when volumes of reciprocal space close to the (311) reflection were explored, no additional Bragg reflections were detected.

One of the molecular stacks present in the single crystal structure adopts an orthogonal orientation with respect to its four neighbouring stacks (see ESI $\dagger$ ). This interaction is characterized by one of the phenyl rings protruding into the 'bay' area between the phenyl rings of an adjacent molecule. If one of the molecular stacks was oriented to produce flat-lying molecular orientation and the herringbone packing arrangement was conserved the molecules in the other stack would stand almost upright with respect to the surface. In this situation the overall out-of-plane orientation of the FePc film is modified but not all of the molecules adopt the flat-lying orientation with respect to the surface. In this case a larger (edge-to-edge rather than faceto-face) out-of-surface-plane intermolecular spacing may be expected. Another possibility is that the crystal structure was altered by the CuI layer and the 'standing' molecular stacks are no longer present in the thin film. Although suggestions of this kind have been made in previous papers concerning structural templating of Pcs, ${ }^{25}$ no experimental proof has been published. In either case the overall out-of-plane orientation of the film was still completely altered by the insertion of the CuI layer as shown by our XRD patterns.

\section{Experimental}

Copper iodide (Sigma Aldrich, UK) was used as received and evaporated from a home built evaporator at $340{ }^{\circ} \mathrm{C}$ at a rate of $0.5 \AA \mathrm{s}^{-1}$ as measured by a calibrated quartz crystal microbalance. FePc (Sigma Aldrich, UK) was triply purified by thermal gradient sublimation ${ }^{16}$ and the resulting crystals were used for growth from a homebuilt evaporator at $375{ }^{\circ} \mathrm{C}$ at a rate of $0.2 \AA \mathrm{s}^{-1}$.

All films were grown in a custom built ultra-high vacuum (UHV) chamber with a base pressure of $3 \times 10^{-9} \mathrm{mbar}$ in which organic and inorganic materials were sublimed onto ambient and elevated temperature substrates. The substrate temperature was measured using a K-type thermocouple mounted close to the sample and calibrated using an optical pyrometer. Substrates were $10 \times 10 \mathrm{~mm}$ pieces of thermally oxidised silicon (100) single crystal (IDB technologies, UK) cleaned in acetone, Decon-90®-de-ionised water mix and isopropanol. These were dried in a stream of dry nitrogen and UV-ozone cleaned before being loaded into vacuum. 
For single crystal X-ray diffraction (XRD) measurements, crystals were grown using a homebuilt thermal gradient sublimation apparatus with a base pressure of $2 \times 10^{-7}$ mbar. A suitable crystal was selected and mounted on a Mitigen loop with silicon oil on an Oxford Diffraction Xcalibur Gemini diffractometer, equipped with a Ruby CCD area detector, and was held at $293 \mathrm{~K}$ during data collection. Using Olex $2,{ }^{17}$ the structure was solved with the ShelXS ${ }^{18}$ structure solution program using direct methods and refined with the ShelXL ${ }^{18}$ refinement package using least squares minimisation. Thin film XRD patterns were obtained using a Panalytical X'Pert Pro MRD diffractometer with monochromatic $\mathrm{Cu} \mathrm{K} \alpha_{1}$ radiation.

Atomic force microscopy (AFM) images were recorded using an Asylum research MFP-3D in AC mode (tapping mode) using Olympus AC240-TS silicon tips.

\section{Conclusions}

We have prepared and characterised CuI thin film templates and demonstrated morphological control using elevated substrate temperatures during growth. The films were highly crystalline with the (111) faces of individual grains parallel to the plane of the $\mathrm{SiO}_{2}$ surface, and this structure was unchanged with growth temperature. Although polycrystalline films were produced the large grain sizes of the crystallites allows their use as model (111) aligned crystalline substrates. These thin films have been employed to control the molecular orientation and grain size of an evaporated FePc layer concomitantly. Nucleation and growth of thin FePc films was strongly affected by the morphology and structure of the underlying $\mathrm{CuI}$ layer with single CuI (111) faces seemingly promoting FePc (311) domain growth. Finally, redetermination of the crystal structure of FePc from single crystal XRD measurements has allowed accurate assignment of the structural changes observed in these bilayers. This methodology shows promise for preferentially aligning phthalocyanine molecules for use in organic electronics devices and producing model systems to study the interactions at organic/inorganic interfaces.

\section{Acknowledgements}

LAR and TSJ acknowledge support from the Engineering and Physical Sciences Research Council, UK (Grant no. EP/H021388/1). DSK thanks the Science City Research Alliance and the HEFCE strategic development fund for financial support. The Panalytical ${ }^{\circledR}$ MRD diffractometer used in this research was obtained through the Science City Advanced Materials Project: Creating and Characterizing Next Generation Advanced Materials, with support from Advantage West Midlands (AWM) and part funded by the European Regional Development Fund (ERDF).

\section{References}

1 S. Heutz, R. Cloots and T. S. Jones, Appl. Phys. Lett., 2000, 77, 3938.
2 S. M. Bayliss, S. Heutz, G. Rumbles and T. S. Jones, Phys. Chem. Chem. Phys., 1999, 1, 3673-3676.

3 W. Wu, L. A. Rochford, S. Felton, Z. Wu, J. L. Yang, S. Heutz, G. Aeppli, T. S. Jones, N. M. Harrison and A. J. Fisher, J. Appl. Phys., 2013, 113, 013914.

4 P. Sullivan, T. S. Jones, A. J. Ferguson and S. Heutz, Appl. Phys. Lett., 2007, 91, 233114.

5 L. Wang, G. Liu, H. Wang, D. Song, B. Yu and D. Yan, Appl. Phys. Lett., 2007, 91, 063511.

6 S. Heutz, C. Mitra, W. Wu, A. J. Fisher, A. Kerridge, M. Stoneham, A. H. Harker, J. Gardener, H.-H. Tseng, T. S. Jones, C. Renner and G. Aeppli, Adv. Mater., 2007, 19, 3618-3622.

7 C. H. Cheng, J. Wang, G. T. Du, S. H. Shi, Z. J. Du, Z. Q. Fan, J. M. Bian and M. S. Wang, Appl. Phys. Lett., 2010, 97, 083305.

8 T.-M. Kim, H. J. Kim, H.-S. Shim, M.-S. Choi, J. W. Kim and J.-J. Kim, J. Mater. Chem. A, 2014, 2, 8730-8735.

9 B. P. Rand, D. Cheyns, K. Vasseur, N. C. Giebink, S. Mothy, Y. Yi, V. Coropceanu, D. Beljonne, J. Cornil, J.-L. Brédas and J. Genoe, Adv. Funct. Mater., 2012, 22, 2987-2995.

10 I. Hancox, P. Sullivan, K. V. Chauhan, N. Beaumont, L. a. Rochford, R. A. Hatton and T. S. Jones, Org. Electron., 2010, 11, 2019-2025.

11 A. Hoshino, Y. Takenaka and H. Miyaji, Acta Crystallogr., Sect. B: Struct. Sci., 2003, 59, 393-403.

12 C. J. Brown, J. Chem. Soc. A, 1968, 2488.

13 R. W. I. de Boer, M. E. Gershenson, A. F. Morpurgo and V. Podzorov, Phys. Status Solidi A, 2004, 201, 1302-1331.

14 K. Xiao, Y. Liu, G. Yu and D. Zhu, Appl. Phys. A: Mater. Sci. Process., 2003, 77, 367-370.

15 F. Pan, H. Tian, X. Qian, L. Huang, Y. Geng and D. Yan, Org. Electron., 2011, 12, 1358-1363.

16 A. R. McGhie, A. F. Garito and A. J. Heeger, J. Cryst. Growth, 1974, 22, 295-297.

17 O. V. Dolomanov, L. J. Bourhis, R. J. Gildea, J. A. K. Howard and H. Puschmann, J. Appl. Crystallogr., 2009, 42, 339-341.

18 G. M. Sheldrick, Acta Crystallogr., Sect. A: Found. Crystallogr., 2008, 64, 112-122.

19 H.-S. Shim, H. J. Kim, J. W. Kim, S.-Y. Kim, W.-I. Jeong, T.-M. Kim and J.-J. Kim, J. Mater. Chem., 2012, 22, 9077.

20 S. R. Forrest, Chem. Rev., 1997, 97, 1793-1896.

21 D. E. Hooks, T. Fritz and M. D. Ward, Adv. Mater., 2001, 13, 227-241.

22 J. E. S. Kim, E. Lim, K. Lee, D. Cha and B. Friedman, Appl. Surf. Sci., 2003, 205, 274-279.

23 C. Miller, A. Sharoni, G. Liu, C. Colesniuc, B. Fruhberger and I. Schuller, Phys. Rev. B: Condens. Matter Mater. Phys., 2005, 72, 104113.

24 J. Kirner, W. Dow and W. Scheidt, Inorg. Chem., 1976, 15, 1685-1690.

25 S. W. Cho, A. DeMasi, A. R. H. Preston, K. E. Smith, L. F. J. Piper, K. V. Chauhan and T. S. Jones, Appl. Phys. Lett., 2012, 100, 263302. 